Vernez, D.; Milon, A.; Francioli, L.; Bulliard, J.L.; Vuilleumier, L.; Moccozet, L. A numeric model to simulate solar individual ultraviolet exposure. Photochemistry and Photobiology, 87(3):721-728, 2011.

\begin{tabular}{|l|l|}
\hline Author's Postprint version & Final draft post-refereeing \\
\hline Journal website & http://onlinelibrary.wiley.com/journal/10.1111/(ISSN)1751-1097/issues \\
\hline Pubmed link & $\underline{\text { http://www.ncbi.nlm.nih.gov/pubmed/21223287 }}$ \\
\hline DOI & $\underline{10.1111 / \text { j.1751-1097.2011.00895.x }}$ \\
\hline
\end{tabular}




\section{A Numeric Model to Simulate Solar Individual Ultraviolet Exposure}

David Vernez ${ }^{1 *}$, Antoine Milon ${ }^{1}$, Laurent Francioli3 ${ }^{3}$ Jean-Luc Bulliard² ${ }^{2}$ Laurent $V u i l l e u m i e r^{4}$, Laurent Moccozet ${ }^{3}$

${ }^{1}$ Institute of Work and Health (IST), Lausanne, Switzerland.

${ }^{2}$ Cancer Epidemiology Unit, University Institute of Social and Preventive Medicine (IUMSP), Centre Hospitalier Universitaire Vaudois and University of Lausanne,

Lausanne, Switzerland

3Department of Information Systems, Faculty of Social and Economic Sciences, University of Geneva, Switzerland.

4Federal Office of Meteorology and Climatology, MeteoSwiss, Payerne, Switzerland

Address correspondence to:

David Vernez

Institute of Work and Health (IST) 
Rue du Bugnon 21

1010 Lausanne

Switzerland

Phone: $\quad$ +4121314 $\quad$ 74 51

Fax: $\quad+41213147420$

E-mail: david.vernez@hospvd.ch 


\section{ABSTRACT}

Exposure to solar ultraviolet (UV) light is the main causative factor for skin cancer. UV exposure depends on environmental and individual factors. Individual exposure data remain scarce and development of alternative assessment methods is greatly needed. We developed a model simulating human exposure to solar UV. The model predicts the dose and distribution of UV exposure received on the basis of ground irradiation and morphological data. Standard 3D computer graphics techniques were adapted to develop a rendering engine that estimates the solar exposure of a virtual manikin depicted as a triangle mesh surface. The amount of solar energy received by each triangle was calculated, taking into account reflected, direct and diffuse radiation, and shading from other body parts. Dosimetric measurements $(n=54)$ were conducted in field conditions using a foam manikin as surrogate for an exposed individual. Dosimetric results were compared to the model predictions. The model predicted exposure to solar UV adequately. The symmetric mean absolute percentage error was $13 \%$. Half of the predictions were within $17 \%$ range of the measurements. This model provides a tool to assess outdoor occupational and recreational UV exposures, without necessitating time-consuming individual dosimetry, with numerous potential uses in skin cancer prevention and research. 


\section{INTRODUCTION}

Solar ultraviolet (UV) radiation is the main environmental risk factor for the most common cancer worldwide: skin cancer (1). According to the WHO, excessive sun exposure leads to 60000 premature deaths each year (2). Of these, about 48000 are due to melanoma, the most lethal cutaneous cancer, and 12000 to epithelial skin carcinomas (basal cell carcinoma (BCC) and squamous cell carcinoma (SCC)). With an estimated 10 million new cases of BCC and 2.9 million cases of SCC per year (2) the overall burden of epithelial skin cancer is considerable. The lifelong risk of developing BCC or SCC for a fair skinned child is 20$30 \%$ and $10 \%$, respectively (3).

Affecting relatively young people, melanoma is one of the leading causes of lost productive years. Contrary to melanoma, epithelial skin cancers tend to progress slowly and occur mainly on chronically sun-exposed body sites, which facilitates early detection and explains their much more favorable prognosis (3).

Intermittent sun exposure is the major causal factor for melanoma (4) and probably BCC (5). Indeed, steady rises in skin cancer rates over the last 50 years are consistent with the increase in outdoor leisure activities and holidays in sunny areas, and the temporal changes in clothing favoring exposure of a wider skin surface.

SCC is predominantly induced by chronic (cumulative) sun exposure, leaving outdoor workers, such as agricultural and building workers, at greater risk (6-8). The association between occupational UV exposure and epithelial skin carcinomas, and the causal association between acute recreational exposure and melanoma have partly been postulated from differences in site distribution of skin cancers (9). Although the etiologic importance of the anatomical location for 
cutaneous cancer has been established $(10,11)$, exploitation of this variable remains limited, partly because of the lack of precise sun exposure measurements for various body parts during at-risk activities (12).

Increase in incidence of skin cancers and issues regarding ozone layer depletion have increased public awareness towards UV exposure, and consequently, enhanced the need to have reliable UV irradiation exposure data. Currently, UV ground radiation measurement stations operate worldwide (particularly in the United States, European countries and Australasia) using either broadband detectors, spectro-radiometers or multifilter rotating shadowband radiometers. Ground irradiance estimates are also available from satellite retrievals on a global scale $(13,14)$. However, satellite UV data are the results of radiation transfer modelling considering the atmospheric components detected with the satellite instrumentation. As such, their spatial resolution is limited and the data is valid only for relatively large area. Furthermore their uncertainty is large in cloudy or high albedo situations. Finally, radiation transfer model $(15,16)$ estimates are reliable in clear-sky situations. Ground irradiance is unfortunately a poor predictor of effective individual exposure (17). Individual factors such as posture, orientation to sun, clothing and other sun protective behavior, exposure duration and time strongly affect effective exposure levels (18). Occupational exposures beyond $100 \%$ of the ambient ground irradiance have been measured on some body parts of outdoor workers $(17,19)$. Body posture (ie. standing and sitting positions) has been shown to be determinant in the exposure of anatomical areas (20).

Wide variations in relative body exposure have been reported. According to (21), the human body receives 24 to $61 \%$ of the total ambient ground irradiance, depending on the time of day and time of year. For a given individual and weather condition, exposure of various anatomical sites ranges from 13 to $76 \%$ of the exposure to the vertex of the head (22), and posture and orientation accounted for at least $38 \%$ of the total variance of relative individual exposure. When 
applying a geometric correction for the fact that humans rarely lie on a horizontal plane while outdoors, Pope et al. (23) estimated that people are exposed to less than half the ambient ground irradiance. Even when receiving a moderate fraction of the total ambient ground irradiance (ca. 20\%), fair skinned outdoor workers can be exposed to UV levels exceeding those sufficient to induce a sunburn (24).

Dosimeters, such as photo-electrical captors or photosensitive chemicals/biologicals dosimeters are available to assess individual exposure. Occupational exposure limits proposed by the International Commission on Non-lonizing Radiation Protection (ICNIRP) have been adopted in several countries. However individual exposure is seldom measured. Indeed, dosimetry remains tedious and resource consuming as several dosimeters are required per subject to measure body-specific exposure. Moreover, dosimetric measurements tend to be situation-specific and prone to epidemiological biases, making generalization difficult.

These limitations led to attempts to develop tools to predict individual exposure without necessitating individual dosimetry. Recent efforts have been brought to the application of 3D human modeling for estimating exposure to radiation in general (25-28). They are mostly dedicated to medical applications and artificial sources as exposure to X-rays or IRM. Some models are also specifically applying 3D computer graphics techniques to address UV exposure (29-32). These models usually assess exposure of various body parts by correcting the UV irradiance measured on horizontal surfaces or by measuring UV irradiance on an inclined surface corresponding to the tilt on the body. The ASCARATIS (Angle SCAnning RAdiometer for determination of erythemally weighted irradiance on TILted Surfaces) model is an example $(30,33)$. The surface of a three dimensional (3D) human model was coloured according to irradiation data measured for different angles allowing a direct vizualisation of the results. However, this model does not take into account the occlusion phenomenon or shading from other body parts. Another study used polysulphone film dosimeters on a rotating manikin head to set up exposure ratios for some anatomic locations (31). Currently limited to the 
head, this approach could be extended to assess the exposure of the whole body but this would require more worktime and dosimetric resources. A 3D modelling approach, based on solar trajectory and radiative transfer models has also been proposed (34). This approach is limited by the accuracy of the radiative transfer model and its accuracy has not been tested in field conditions.

We present in this paper a prediction model of individual UV exposure based on existing techniques in 3D rendering, and 3D human modeling. We have developed a specific 3D rendering engine adapted to estimate UV light interactions over a 3D body shape surface. The MakeHuman software (35) provides a state of the art modeling environment of human body based on articulated skeleton techniques (36,37). It is used to create 3D surfaces of various human body morphologies and postures that are then fed into the UV rendering engine. This article describes our model, discusses its performances in field conditions and its potential perspectives for public health, prevention and research on skin cancer. 


\section{MATERIAL AND METHOD}

\section{The SimUVEx model}

Our model, named SimUVEx (Simulating UV Exposure), used general continuous datasets (csv files) of ambient ground irradiance to estimate the dose and anatomical distribution of UV received by exposed individuals (Figure 1). The data, were fed into a solar radiation model in which direct I(t), diffuse $D(t)$, and reflected $R(t)$ (reflection from the ground) components were taken into account separately. Exposure to various anatomical locations was obtained by exposing a 3D virtual manikin to the radiation sphere, discretized into $\mathrm{n}$ sub-surfaces, for a given duration. The exposure levels and doses computed during the simulation can be visualized on comprehensive 3-D images using expressive rendering techniques.

(Figure 1 about here)

\section{Input data}

Three ambient irradiance datasets over time are required as input parameters in the model, including: (1) the direct, (2) the diffuse, and (3) the ground reflected irradiance $\left(\mathrm{W} / \mathrm{m}^{2}\right)$. Sun position, defined by its azimuth $\mathrm{p}(\mathrm{t})$ and zenith $\mathrm{d}(\mathrm{t})$ angles, is also required to describe direct irradiation. The model derives radiances from these input using simplifying hypotheses. Such input data can be obtained from meteorological stations equipped with multiples broadband radiometers 
(e.g. one for direct - or global with subsequent subtraction of the diffuse component - radiation, one for diffuse radiation with a shadowing disc and one turned upside down for reflected radiation). Alternatively these data can be obtained from atmospheric radiation transfer models (RTM) such as libRadtran (15) or TUV (16). In clear-sky situations, RTM programs can give accurate estimates of the irradiance components mentioned above. However, the use of data issued from RTM in a cloudy atmosphere is usually precluded by the complexity of accurately describing the cloudy situation. In response, many efforts have been recently devoted to reconstructing UV irradiance in complex situations based on proxies. Particularly, the overall influence of cloud on radiation can very well be extracted from measurements of the total solar irradiance (not restricted to the UV range) and extrapolated to the UV range by semi-empirical algorithms (for an overview, see (38)). Such methods allow profiting from the much higher density of total solar irradiance observation networks compared to observations restricted to the UV range. However, until now, these methods have been applied to global irradiance and not to the direct and diffuse components, and some more developments will be required for inferring the components from such methods.

Postural and morphological data corresponding to the exposed individuals are also required as input parameter. Postures are defined as a set of angles values. Each value defines the angle at a joint articulation of a simplified skeleton. Morphologies are defined as a set of local scaling values. Each value is applied to locally scale a 3D shape body template. Any posture or morphology can be produced with the MakeHuman software and fed into the simulation. The simulation engine reads $3 \mathrm{D}$ meshes representing the designed body shapes.

Radiation modeling 
Derivation of UV radiation exposure from irradiance is based on simplifying the reflected $R(t)$ and diffuse $D(t)$ components as hemispherical isotropic sources with timedependent intensities (Figure 2a). Such an approach is solved by deriving an isotropic (constant) radiance in a hemisphere from a measured irradiance. For example for diffuse radiation, assuming the broadband detector has a perfect Lambertian (or cosine) response:

$I_{D}(t)=\int_{\varphi=0}^{2 \pi} d \varphi \int_{\theta=0}^{\pi / 2} d \theta u_{D}(\theta, \varphi, t) \cos \theta \sin \theta=2 \pi u_{D}(t) \int_{\theta=0}^{\pi / 2} d \theta \cos \theta \sin \theta=2 \pi u_{D}(t) \frac{1}{2}$

Where $I_{D}(t)$ is the measured diffuse irradiance and $u_{D}(t)$ is the diffuse radiance. Then, the integrated diffuse radiance from a given direction and solid angle covering a given surface area in the hemisphere is:

$D(t)=u_{D}(t) \int d \varphi \int d \theta \sin \theta=u_{D}(t) A_{S}=2 I_{D}(t) A_{S} / 2 \pi$

Both hemispheres were discretized into regular sub-surfaces (typically about 400 sub-surfaces), and the term $A_{S} / 2 \pi$ represents the ratio between the solid angle corresponding to the sub-surface and the hemisphere. Thus, each sub-surface was assigned with one portion of the total radiation component and was represented by its barycenter. The reflection from the ground was assumed to be isotropic, and equal amount of energy was assigned to each sub-surface element of the bottom half-sphere. However, diffuse radiation is known to be anisotropic, particularly near the ground and close to the sun during clear days (39). The model accounted for anisotropy near the ground by attenuating the energy allocated to sub-surfaces at the base of the diffuse half-sphere. The diffuse radiation was assumed to decrease linearly between an elevation angle of $25^{\circ}$ and the horizontal plane $\left(0^{\circ}\right)$. The difference between the latter representation and the former 
hypothesis of diffuse radiance isotropy introduces an uncertainty of a few percent. Anisotropy near the sun was not considered in the model in order to limit its complexity. Anisotropy is sun-position dependent, and a possible inclusion of its effect would require recalculation of each simulation step.

The direct component I $(\mathrm{t})$ is described as a parallel source of radiation varying in intensity with time and in direction with the sun position (Figure 2a).

(Figure 2 about here)

\section{Body modeling}

Traditional 3D human modeling and animation approach, based on articulated skeleton and 3D surface skinning $(36,37,40)$ were used to produce a 3D virtual manikin in standard working positions (Figure 2b). To estimate UV exposure, only the outer shape (skin) of the body was required. Therefore, the surface of the virtual manikin was depicted as a single 3D mesh of connected triangles, whose size density depends on the desired resolution (typically about 4,000 meshes). The virtual manikin models were produced in the open source software modeling package MakeHuman (35), an interactive modeling tool for creating custom 3D human characters.

In order to optimize the computing resources, the density of the vertices in the mesh can be tuned to provide an appropriate balance between body details and computation time. We produced the body mesh at a very high density and apply mesh simplification techniques (41) to reduce the density. These techniques 
reduce the mesh density but keep the reduced mesh shape as close as possible to the original one. A low density mesh loses body shape details such as the nose or the ears but reduces the required computation time. For our experiments, we used a 4000 vertices resolution mesh. It appeared to provide an appropriate detailed mesh at a reasonable time cost.

Radiation-body interaction modeling

Each triangle of the virtual manikin received a specific amount of radiation according to its exposure to the different virtual sources of solar radiation. This exposure depended on radiation intensity, orientations of the triangles and light sources as well as shading from body parts (e.g. the stomach is protected from direct radiation when the sun is behind the body). Figure 3 illustrates the principles of diffuse, reflected and direct radiation exposures computations.

Direct radiation. The direct component was considered as a directional light source I(t). For each time step, we estimated which vertices in the 3D mesh were visible from the directional light source. As illustrated in (Figure 3b), the amount of direct radiation energy $\left(\mathrm{E}_{\mathrm{v}}\right)$ received by a vertex can be expressed as:

$$
\left[\mathrm{J} / \mathrm{m}^{2}\right]
$$

\section{$E_{v}=\int^{t} I(t) \cdot \cos \phi \cdot d t$}

$\phi$ beingtthe angle of incidence between the direct light and the vertex normal (also called pseudo-normal). The vertex normal $\overrightarrow{N_{v}}$ is obtained by averaging and normalizing all the normals of the triangular meshes $\vec{N}_{t}$ adjacent to the vertex: For $n$ adjacent triangular meshes, the vertex normal becomes: 
$\overrightarrow{N_{v}}=\frac{\sum_{t=1}^{n} \vec{N}_{t}}{\left|\sum_{t=1}^{n} \vec{N}_{t}\right|}$

Diffuse and reflected radiation. For each vertex of the 3D mesh, the number of sub-surfaces of each hemisphere visible from the vertex were calculated (see Figure 3 a) to built a so-called "visibility" map. This map needed to be constructed only once for the whole exposure session (only the exposure value changed over time). Visibility computations required numerous intersection estimations, and consequently needed substantial computation time and resources (42). The energy contribution from each visible sub-surface to exposure of a given vertex was computed similarly to the direct radiation, $\phi$ being the angle between the vertex normal and the normal to the diffuse/reflected sub-surface considered.

(Figure 3 about here)

Visualization. The 3D visualization aims at enhancing understanding. To reveal the exposure distribution over the body surface, we defined an intuitive expressive rendering schema. The exposure value computed for each vertex was mapped to a simple three color scale: blue, green, and red for low, 
intermediate, and high values, respectively. Colors represented the ratio of the vertex exposure value to the ambient ground surface dose on a horizontal plane (values higher than the ground surface dose were white).

Implementation

The prototype application was developed in $\mathrm{C}++$, based on available open source library VCG (43), dedicated to manipulation and processing of triangle meshes, as a plug-in in the open source software package MESHLAB (44). The application reads continuous datasets of ambient ground irradiation measurements and sets of $3 \mathrm{D}$ body models representing people in various positions. We defined a simple scenario by assigning any available position to given time frames. The simulation engine estimated the exposure. A dataset of estimation measurements and the visualizations of the exposed 3D manikins were produced.

\section{Field measurements}

The field measurements were mostly collected during a session from 21 to 25.05 .2007 at the MeteoSwiss Payerne station $\left(46.815^{\circ} \mathrm{N}, 6.944^{\circ} \mathrm{E}\right)$, which is located in an open field area at $491 \mathrm{~m}$ above sea level. Some supplementary data were collected at the same location on 10.07.2008. Only data collected between 9am and 6pm were used in the analysis. During the first session, relative sun duration was between 50 and $90 \%$ depending on the day, and 95\% for the 10.07.2008, indicating direct sunlight for most of these days and predominant clear-sky conditions. The Payerne facility is part of the Baseline Surface Radiation Network (BSRN) of the WMO World Climate Research Program (45). Ambient direct, diffuse and reflected UV irradiance are measured concomitantly every minute at this facility. All components are measured with SL501A broadband detectors with filters mimicking the erythemal response. The instrument measuring the direct component is mounted within a 
collimator on a sun tracker, the diffuse component is measured by an instrument shaded with a disc controlled by the sun tracker and the reflected component by an instrument turned upside down. The UV broadband radiometers used at the Payerne station undergo strict quality assurance procedures including regular calibrations traceable to the European Ultraviolet Calibration Center (46).

We used CIE erythemaly weighted Spore film dosimeters (BioSense, Bornheim, Germany) positioned on an articulated foam manikin (Etal'Pro, Annecy, France) for individual exposure measurements. The lower detection limit of the dosimeters used was $100 \mathrm{~J} \cdot \mathrm{m}^{-2}$ (1SED) with accuracy ranging from $5 \%$ to $20 \%$ standard deviation (SD, laboratory conditions and unfavorable field conditions, respectively) $(47,48)$. A SD of $15 \%$, corresponding to conservative average accuracy in field conditions has been considered in this paper. Five static body postures were investigated: seated, kneeling, standing bowing, standing erect arms down, and standing erect arms up. Eight body locations were investigated systematically for each posture: neck, low back, left and right shoulders, right wrist, chest, forehead, and top head. Top head exposure was represented by dosimeters positioned on a horizontal plane. Additional body locations were also investigated for the standing erect arms down posture, which is common to numerous leisure and occupational activities. In total, 54 dosimeters were used.

\section{Numeric simulations}

First, simulation runs were conducted to predict the exposure of a plane surface at ground level. This scenario mimics the exposure of the broadband detector on a horizontal plane. Direct, diffuse and reflected radiation were computed and measured separately every minute. Simulation results were then compared to the radiometers measurements to validate some basic model elements (e.g. radiation model, radiation-surface algorithm) and identify possible modeling errors (later referred to as internal validation). 
Secondly, simulation runs were conducted to predict the exposure of the virtual 3D manikin in the same body posture as the foam manikin. Discrete computations, using time steps of 1 minute, were performed successfully to simulate daily exposure. Simulation times to compute a single day exposure in these conditions (9 hrs, 1 min step, 540 simulation steps, 4000 meshes) using a personal computer (HP EliteBook 8540p, 2.4 MHz, 4 Gb RAM) were typically of 8 min when starting from scratch or 3 min when using pre-existing visibility maps.

The computed daily doses for the 5 investigated body postures and 8 body locations were compared to dosimetric measurements obtained in field conditions. This comparison was used to test the performance of the overall model (field validation).

The performance of the SimUVEx model was evaluated in terms of the symmetric mean absolute percentage error (sMAPE) as follows.

$\operatorname{sMAPE}=\frac{1}{\mathrm{n}} \cdot \sum_{\mathrm{i}=1}^{\mathrm{n}=1} \frac{\left|\mathrm{e}_{\text {meas }}-\mathrm{e}_{\text {pred }}\right|}{\left(\mathrm{e}_{\text {meas }}|+| \mathrm{e}_{\text {pred }} \mid\right)} \quad$ Eq. 3

Where $\mathrm{n}$ is the number of measurements, $e_{\text {meas }}$ the measured exposure and $e_{\text {pred }}$ the predicted exposure. sMAPE ranges between $0-100 \%, 0 \%$ corresponding to a perfect prediction. 


\section{RESULTS AND DISCUSSION}

\section{Model validation}

The SimUVEx model predicted accurately the measured values during internal validation. Relative errors in global irradiation of $3-4 \%$ were typically found between simulated plane surface and the ground measurements during an exposure day. SMAPE were $3 \%, 1 \cdot 10^{-6} \%, 2 \cdot 10^{-2} \%(n=482)$, for diffuse, direct, and reflected components, respectively. The accuracy of the direct and reflected prediction is partly explained by the fact that the same measurements were used as field reference values and as input data in the setting of the light source model during internal validation. This procedure excludes de facto any interference from field measurements uncertainties. These results indicate that: (1) the hypotheses used in the light source model are adequate (e.g discretized diffuse and reflective half-spheres) and that (2) there was no major flaw in the computation method and algorithm used. The lower performance observed for the diffuse irradiation prediction is due to the fact that the diffuse component was considered as an isotropic model input, but was allowed to decrease linearly to 0 at low elevation angles (between a threshold angle and the horizon) in the computation. Overall the impact of this simplifying hypothesis on the performance of the model remains small.

The results of the field validation obtained for various body postures and body locations are presented in Figure 4. SimUVEx predictions were in the same order of magnitude as the measured values and overall in agreement with the expected values (gray line). Five measurements were removed; three were aberrant when compared to other measurements collected the same day and were obvious dosimetric errors; and two were irrelevant because of an inadequate 
positioning of the foam manikin's head during the dosimetric measurements. The SMAPE for the remaining results $(n=49)$ was $13 \%$. Half of the predictions fell within a $17 \%$ range of the measurements and $75 \%$ within a $40 \%$ range of the measurements.

(Figure 4 about here)

Several sources of uncertainties may explain the differences observed between predicted and measured values in field conditions: (1) the model hypothesis, (2) the accuracy of the spore film dosimeters and, (3) the field measurement procedure. It was difficult to have an exact match between the posture and morphology of the foam and virtual manikins. This may lead to discrepancies in both orientation and shading of the body surfaces. Moreover, due to their shape and size, dosimeters may have a slightly different orientation compared to body surfaces. Uncertainties related to the measurement procedure were not accounted for in the $15 \%$ dosimetric error but may contribute significantly to the overall uncertainty. Uncertainties also arise from the model radiance derived from irradiance measurements. Careful and regular calibration were made for reducing the irradiance uncertainty due to differences between the shape of the broadband radiometer filter transfer function and the erythemal weighting function, as well as filter degradation and non-Lambertian response. Uncertainty for 1-min measurements is on the order of $10 \%$. Part of it is statistical variation and is reduced when considering longer aggregation times, but a $5-8 \%$ uncertainty remains from calibrating procedures (49). 
Predictions/measurements ratios by body parts are presented in Figure 5. Overestimated and underestimated predictions appear overall balanced, showing no systematic bias in the model. An underestimation of the predictions can however, be observed in Figure 4 for high exposure measurements. On average, predictions were $27 \%$ lower than actual measurements but only for measurements over $3000 \mathrm{~J} / \mathrm{m}^{2}$. The fact that anisotropy near the sun was not taken into account in the model may explain this tendency. Highly exposed body surfaces were facing the sun and were therefore more affected by this phenomenon. Measurement conditions reflected a worst-case scenario in that respect as anisotropic effects are more important in clear-sky conditions (39).

Although site-specific data were limited, no clear pattern emerged when considering body parts. This suggests that random errors (e.g. dosimeter orientation error) play a major role in the overall uncertainty. The contribution from uncertainties in measurement procedures is unknown; however, the overall model to observation differences remain low (in the context of a deterministic model), indicating that the model was adequate in predicting exposure to solar UV.

(Figure 5 about here)

\section{Using simulation data}


Simulation produced large amount of data since direct, diffuse and reflected radiation were computed separately for each vertex at each simulation step. Depending on the needs, SimUVEx can compute and provide these data in several ways: average exposure doses for various body parts, doses for specific anatomic locations, instantaneous exposures, or relative contributions of different radiation sources. Simulation results can be visualized, using false color mapping (Figure 6a-c).

(Figure 6 about here)

Body posture and orientation to the sun play a key role in skin exposure to UV and are duly taken into account in the model. In real life situations, individuals hardly stay static and postural and orientation changes over the exposure period must be considered. Thus, additional functionalities were integrated into the numeric tool. Two dynamic situations were considered: a changing orientation for a given posture and a sequence of different orientations and postures. The first situation is typical for an activity involving movements with a predominant posture (e.g. working in a vineyard, on a tractor). The virtual manikin was rotated in each simulation step to obtain an "average" orientation (Figure $6 \mathrm{~d}$ ). In the second situation, orientation and posture were defined over time by the user. This method was more comprehensive and required a sequential recording of the postural activity for each exposed individual.

\section{Perspectives}


SimUVEx is an inexpensive tool to assess occupational and recreational exposure to solar radiation, providing exposure estimates and detailed exposure distribution for the entire body. Skin cancer research and prevention may benefit from this tool in identifying overexposed populations due to outdoor activities in various environmental conditions, and thus trigger preventive actions. It must be pointed out that the model requires no individual exposure (dosimetric) data. Only postural information and ambient ground irradiance data (sun location and diffuse, reflected and direct UV irradiance), which may be obtained from meteorological stations equipped with broadband UV radiometers, are required.

However the most common measurement of UV irradiance is global UV irradiance, while the direct and diffuse components are only available at a limited number of stations. Alternatively to broadband UV radiometry, other data sources can enhance the input data availability to virtually any location: satellite estimate, radiation transfer modelling or semi-empirical UV reconstruction methods. Satellite estimates and radiation transfer models are reliable in clear-sky situations but significant uncertainties arise in more complex situations. This limitation is acceptable from a public health perspective since clear-sky situations usually lead to the most important doses. Semi-empirical methods perform well in cloudy situations, but have been applied to global irradiance rather than to the direct and diffuse components. Since new pyranometers have been developed that can provide the components without costly sun tracking systems (Delta-T pyranometer - SPN1), it would probably be possible to devise semi-empirical methods for the direct and diffuse component as well. In situ measurements (ground irradiance measurements), using portable dosimeters can also be considered when facing specific environmental conditions (e.g. reduced sky view). 
In retrospective epidemiologic studies, this tool can help quantifying past UV exposures, producing an exposure-response, and ultimately provides a better understanding of skin cancer risk. Moreover, the distribution of anatomical UV exposure can be compared with epidemiological data on site of skin cancers and enhance our etiologic understanding.

In prospective exposure assessment studies, simulations may be performed to prevent overexposures in new activities or to assess the impact of new environmental conditions or the effectiveness of individual protection measures (eg. protective clothing) on UV dose received. Acute or subacute exposure can be investigated using simulation of short time periods or instantaneous exposure levels. Short-term dosimetry data are sparse despite the fact that acute and subacute exposures are known to contribute significantly to melanoma risk (18).

A great advantage of this tool is that it can readily illustrate exposure scenarios to various audiences (researchers, health authorities, work employees, the general public). This is especially relevant in the prevention of UV overexposure (e.g. by helping people visualize the impact of UV radiation (red, green, and blue) and encourage them to dress adequately for outdoor activities. Sensitizing people about the importance of their own activity in reducing exposure has been shown to be an effective strategy (50). The vizualisation tool could serve to illustrate the effect of some behavioral changes on the UV dose received or to translate UV irradiation information, such as the UV index, into sunburn risk for typical daily activities.

The current SimUVEx version allows the incorporation of clothes and sunscreens with their protection index to different body parts. Further developments to enlarge the tool capabilities are also being considered. New body postures could be added to the five postures considered in this study to target a wider range of occupational or leisure activities (e.g, lying on the ground when sunbathing). New morphologies could also be added to the tool libraries to address populations 
of different size, gender or age (e.g. UV exposure to children or women). 3D objects can also be added in the model to take into account sources of shade specific to the local environment (e.g. building, parasol). It could be used to measure the personal solar UV exposures in diffuse UV settings for instance.

SimUVEx may also be refined to improve its accuracy. Diffuse or reflected half-sphere description could be enhanced by adding more sub-surfaces or taking into account anisotropy near the sun. However, the benefit (accuracy) of such development has to be carefully weighed against its cost (computation time). We believe the current model is a good compromise between these two factors. Further developments may be nevertheless of interest in unusual environmental exposure conditions, such as highly reflecting and heterogeneous environment (e.g. water surface). 


\section{REFERENCES}

1. World Health Organization (WHO) (1992) Solar and Ultraviolet Radiation. In IARC Monographs on the evaluation of carcinogenic risks to humans.(Edited by World Health Organization), London.

2. Lucas, R., T. McMichael, and W. Smith (2006) Solar ultraviolet radiation : Global burden of disease from solar ultraviolet radiation. World Health Organization, Geneva.

3. Bulliard, J. L., Panizzon R.G., and F. Levi (2009) Epidemiology of epithelial skin cancers. Rev Med Suisse 5, 882-888.

4. Elwood, J. M. and J. Jopson (1997) Melanoma and sun exposure: an overview of published studies. Int J Cancer 73, 198-203.

5. Kricker, A., B. K. Armstrong, and D. R. English (1995) Does intermittent sun exposure cause basal cell carcinoma? a case-control study in Western Australia. Int J Cancer 60, 489-494.

6. Armstrong, B. K. and A. Kricker (2001) The epidemiology of UV induced skin cancer. J Photochem Photobiol B 63, 8-18.

7. Levi, F., G. Erler, V. C. Te, L. Randimbison, and C. La Vecchia (2001) Trends in skin cancer incidence in Neuchâtel, 1976-98. Tumori 87, 288-289.

8. Levi, F., V. C. Te, L. Randimbison, G. Erler, and C. La Vecchia (2001) Trends in skin cancer incidence in Vaud: an update, 1976-1998. Eur J Cancer Prev 10, 371-373.

9. Beral, V. and N. Robinson (1981) The relationship of malignant melanoma, basal and squamous skin cancers to indoor and outdoor work. $\mathrm{Br} \mathrm{J}$ Cancer $44,886-891$.

10. Bulliard, J. L., B. Cox, and J. M. Elwood (1997) Comparison of the site distribution of melanoma in New Zealand and Canada. Int J Cancer 72, 231-235.

11. Franceschi, S., F. Levi, L. Randimbison, and C. La Vecchia (1996) Site distribution of different types of skin cancer: new aetiological clues. Int J Cancer 66, 1-5.

12. Bulliard, J. L., D. De Weck, T. Fisch, A. Bordoni, and F. Levi (2007) Detailed site distribution of melanoma and sunlight exposure: aetiological patterns from a Swiss series. Ann Oncol 18, 789-794. 
13. van Geffen, J., van der A, M., van Weele, M., Allaart, M., and Eskes, H. Surface UV radiation monitoring based on GOME and SCIAMACHY. 572. 2005. Salzburg, ESA publication . Proceedings of the ENVISAT \& ERS Symposium. 6-9-2004.

14. Eck, T. F., P. K. Bhartia, and J. B. Kerr (1995) Satellite estimation of spectral UVB irradiance using TOMS derived total ozone and UV reflectivity. Geophys. Res. Lett. $22,611-614$

15. Mayer, B. and A. Kylling (2005) Technical note: The libRadtran software package for radiative transfer calculations - description and examples of use. Atmospheric Chemistry and Physics 5, 1855-1877.

16. Madronich, S. (1998) The TUV software package version 4.1a. Available at: ftp://acd.ucar.edu/user/sasha. Accessed on 24-6-2010.

17. Milon, A., P. E. Sottas, J. L. Bulliard, and D. Vernez (2007) Effective exposure to solar UV in building workers: influence of local and individual factors. J. Expo. Sci. Environ. Epidemiol. 17, 58-68.

18. Autier, P., M. Boniol, and J.-F. Doré (2007) Sunscreen use and increased duration of intentional sun exposure: Still a burning issue. International Journal of Cancer 121, $1-5$.

19. Gies, P. and J. Wright (2003) Measured Solar Ultraviolet Radiation Exposures of Outdoor Workers in Queensland in the Building and Construction Industry. Photochem Photobiol 78, 342-348.

20. Parisi, A. V., M. G. Kimlin, R. Lester, and D. Turnbull (2003) Lower body anatomical distribution of solar ultraviolet radiation on the human form in standing and sitting postures. J Photochem Photobiol B 69, 1-6.

21. Parisi, A. V., M. G. Kimlin, J. C. Wong, and R. A. Fleming (1996) The effects of body size and orientation on ultraviolet radiation exposure. Photodermatol Photoimmunol Photomed 12, 66-72.

22. Wright, C., R. Diab, and B. Martincigh (2004) Anatomical distribution of ultraviolet solar radiation. South Afr J Sci 100, 498-500.

23. Pope, S. J. and D. E. Godar (2010) Solar UV geometric conversion factors: horizontal plane to cylinder model. Photochem Photobiol 86, 457-466.

24. Hammond, V., A. I. Reeder, and A. Gray (2009) Patterns of real-time occupational ultraviolet radiation exposure among a sample of outdoor workers in New Zealand. Public Health 123, 182-187. 
25. Xu XG, Taranenko V, Zhang J, and Shi C (2007) A boundary-representation method for designing whole-body radiation dosimetry models: pregnant females at the ends of three gestational periods-RPI-P3, -P6 and -P9. Phys Med Biol 52, 7023-7044.

26. Christ, A., W. Kainz, E. G. Hahn, K. Honegger, M. Zefferer, E. Neufeld, W. Rasher, R. Janka, W. Bautz, J. Chen, B. Kiefer, P. Schmitt, H. Hollenbach, J. Shen, M. Oberle, D. Szczerba, A. Kam, J. W. Guag, and N. Kuster (2010) The Virtual Family-development of surface-based anatomical models of two adults and two children for dosimetric simulations. Phys Med Biol 55, 23-28.

27. Cassola, V. F., V. J. de Melo Lima, R. Kramer, and H. J. Khoury (2010) FASH and MASH: female and male adult human phantoms based on polygon mesh surfaces. Phys Med Biol 55, 133-162.

28. Zhang, J., Y. H. Na, P. F. Caracappa, and X. G. Xu (2009) RPI-AM and RPI-AF, a pair of mesh-based, size-adjustable adult male and female computational phantoms using ICRP-89 parameters and their calculations for organ doses from monoenergetic photon beams. Phys Med Biol 54, 5885-5908.

29. Oppenrieder, A., P. Hoeppe, and P. Koepke (2004) Routine measurement of erythemally effective UV irradiance on inclined surfaces. Journal of Photochemistry and Photobiology B: Biology 74, 85-94.

30. Hoeppe, P., A. Oppenrieder, C. Erianto, P. Koepke, J. Reuder, M. Seefeldner, and D. Nowak (2004) Visualization of UV exposure of the human body based on data from a scanning UV-measuring system. Int J Biometerol 49, 18-25.

31. Kimlin, M. G., A. V. Parisi, and N. D. Downs (2003) Human UVA exposures estimated from ambient UVA measurements. Photochem Photobiol Sci 2, 365-369.

32. Streicher, J. J., W. C. Culverhouse, M. S. Dulberg, and R. J. Fornaro (2004) Modeling the anatomical distribution of sunlight. Photochem Photobiol $79,40-47$.

33. Oppenrieder, A., P. Hoeppe, and P. Koepke (2004) Routine measurement of erythemally effective UV irradiance on inclined surfaces. J Photochem Photobiol B 74, 8594.

34. Streicher, J. J., W. C. Culverhouse, M. S. Dulberg, and R. J. Fornaro (2004) Modeling the Anatomical Distribution of Sunlight. Photochem Photobiol 79, $40-47$.

35. MakeHuman (2010) Open source tool for making 3D characters. Available at: http://www.makehuman.org/. Accessed on 25-6-2010.

36. Di Giacomo, T., H. Kim, L. Moccozet, and N. Magnenat-Thalmann (2007) Control structure and multi-resolution techniques for virtual human representation. In Shape Analysis and Structuring.(Edited by L. De Floriani and M. Spagunolo), Springer Verlag, Berlin. 
37. Di Giacomo, T., L. Moccozet, N. Magnenat-Thalmann, R. Boulic, and D. Thalmann (2007) Automatic character skeletonization and deformation, State of the Art Report (STAR). In Annual Conference of the European Association for Computer Graphics (Eurographics 2007).

38. Koepke, P., Backer, H. D., Bais, A., Curylo, A., Eerme, K., Feister, U., Johnsen, B., Junk, J., Kazantzidis, A., Krzyscin, J., Lindfors, A., Olseth, J. A., den Outer, P., Pribullova, A., Schmalwieser, A. W., Slaper, H., Staiger, H., Verdebout, J., Vuilleumier, L., and Weihs, P. Modelling solar UV radiation in the past: comparison of algorithms and input data. Slusser, J. R. and Comerón, A. 6362. 2006. Proc. SPIE. Remote Sensing of Clouds and the Atmosphere XI. 2006.

39. Kuchinke, C. and M. Nunez (2003) An anisotropy correction method for all-sky measurements of diffuse UV-B erythemal irradiance. J Atmos and Ocean Technol 20, 1253-1533.

40. Magnenat-Thalmann, N. and D. Thalmann (2004) Handbook of virtual humans. J. Wiley, Chichester.

41. Luebke, D., M. Reddy, J. D. Cohen, A. Varshney, B. Watson, and R. Huebner (2003) In Mesh Simplification, Level of Detail for 3D Graphics. pp. 19-46. Morgan Kaufmann, San Francisco.

42. Bittner J and Wonka P (2003) Visibility in Computer Graphics, Environment and Planning B. Planning and Design 30, 729-756.

43. VCG (Visualization and Computer Graphics) (2010) VCGLib. Available at: http://vcg.sourceforge.net. Accessed on 24-6-2010.

44. Meshlab (2010) Open source for the processing and editing of unstructured 3D triangular meshes. Available at: http://meshlab.sourceforge.net/. Accessed on 24-62010.

45. Ohmura, A. (1998) Baseline Surface Radiation Network (BSRN): New precision radiometry for climate research. Bull. Am. Meteorol. Soc. 79, $2115-2136$.

46. Hülsen, G. and J. Gröbner (2007) Characterization and calibration of ultraviolet broadband radiometers measuring erythemally weighted irradiance. Appl. Opt. 46, 58775886 .

47. Moehrle, M., M. Korn, and C. Garbe (2000) Bacillus subtilis spore film dosimeters in personal dosimetry for occupational solar ultraviolet exposure. Int. Arch. Occup. Environ. Health 73, 575-580.

48. Furusawa, Y., L. E. Quintern, H. Holtschmidt, P. Koepke, and M. Saito (1998) Determination of erythema-effective solar radiation in Japan and Germany with a spore monolayer film optimised for the detection of UVB and UVA - results of a field campaign. Appl Microbiol Biotechnol 50, 597-603.

49. Walker, D. (2009) Cloud effects on erythemal UV radiation in a complex topography. PhD Thesis $n^{\circ} 18415$, ETH, Zurich. 
50. Kimlin, M. G. and A. V. Parisi (2001) Usage of real-time ultraviolet radiation data to modify the daily erythemal exposure of primary schoolchildren. Photodermatol Photoimmunol Photomed 17, 130-135. 


\section{ACKNOWLEDGEMENTS:}

This research was supported by the workers and work relations protection office of Valais (SPT), the Swiss National Accident Insurance Organization (SUVA) and the French Agency for Environmental and Occupational Health Safety (Afsset) 


\section{FIGURE CAPTIONS:}

Figure 1. Schematic view of the SimUVEx model

Figure 2. Model components (A) irradiation model including direct, diffuse and reflected components, (B) Virtual manikins of 4000 and 16 '000 meshes

Figure 3. Light-body interaction modeling (A) example of a visibility map used in the case of diffuse or reflected irradiation, (B) computing the energy received by a vertex for a given light ray

Figure 4. Measured vs. predicted daily exposure doses to solar UV ( $n=54)$. Horizontal segments illustrate the $15 \%$ uncertainty on dosimetry. Hollow dots are outliers identified as measurement errors. 
Figure 5. Predicted/Measured daily exposure doses to solar UV ( $n=49)$. Shaded area illustrates the dosimetric uncertainty (15\% relative error). Body parts are illustrated as such: $(\odot)$ neck, $(\square)$ low back, $(\Delta)$ left shoulder $(\diamond)$ right shoulder, $(\Delta)$ right wrist, $(\diamond)$ forehead, $(\square)$ top head, $(\Theta)$ chest and $(+)$ other body location.

Figure 6. Visualizing simulation results for a daily exposure (A) standing erect arms down (B) kneeling (C) seated (D) seated with a changing orientation $\left(90^{\circ}\right.$ rotation every $30 \mathrm{~min}$.). Blue, green, red and white colors were attributed for $0-39 \%, 40-78 \%, 79-100 \%$ and $>100 \%$ of the ground irradiance respectively. 
Figure1. Schematic view of the SimUVEx model 


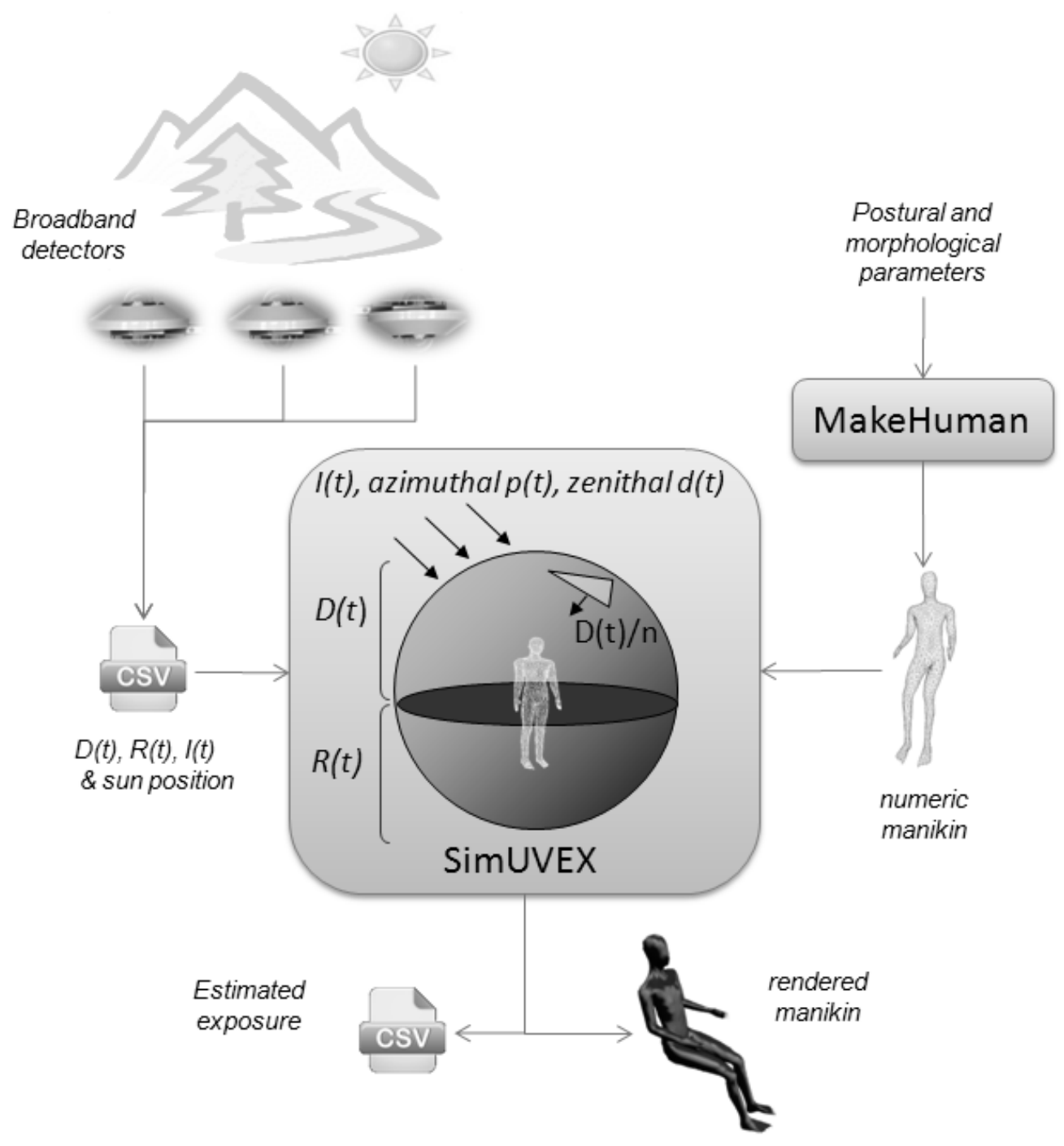


Figure 2. Model components (A) irradiation model including direct, diffuse and albedo sources, (B) Virtual manikin of 4000 and $16{ }^{\prime} 000$ meshes 
$I(t)$, azimuthal $p(t)$, zenithal $d(t)$
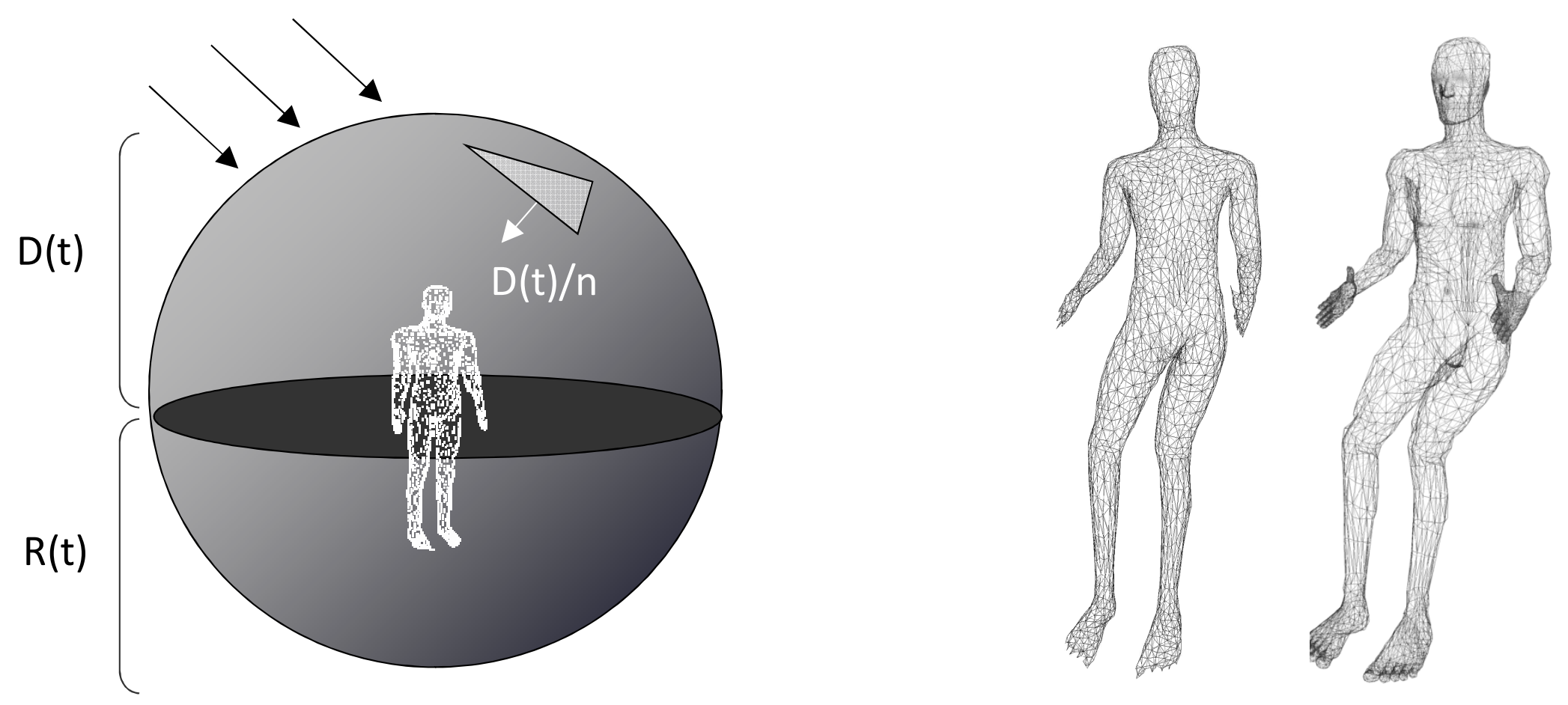
Figure 3. Light-body interaction modeling (A) example of a visibility map used in the case of diffuse irradiation or albedo, (B) computing the energy received by a vertex for a given light ray 

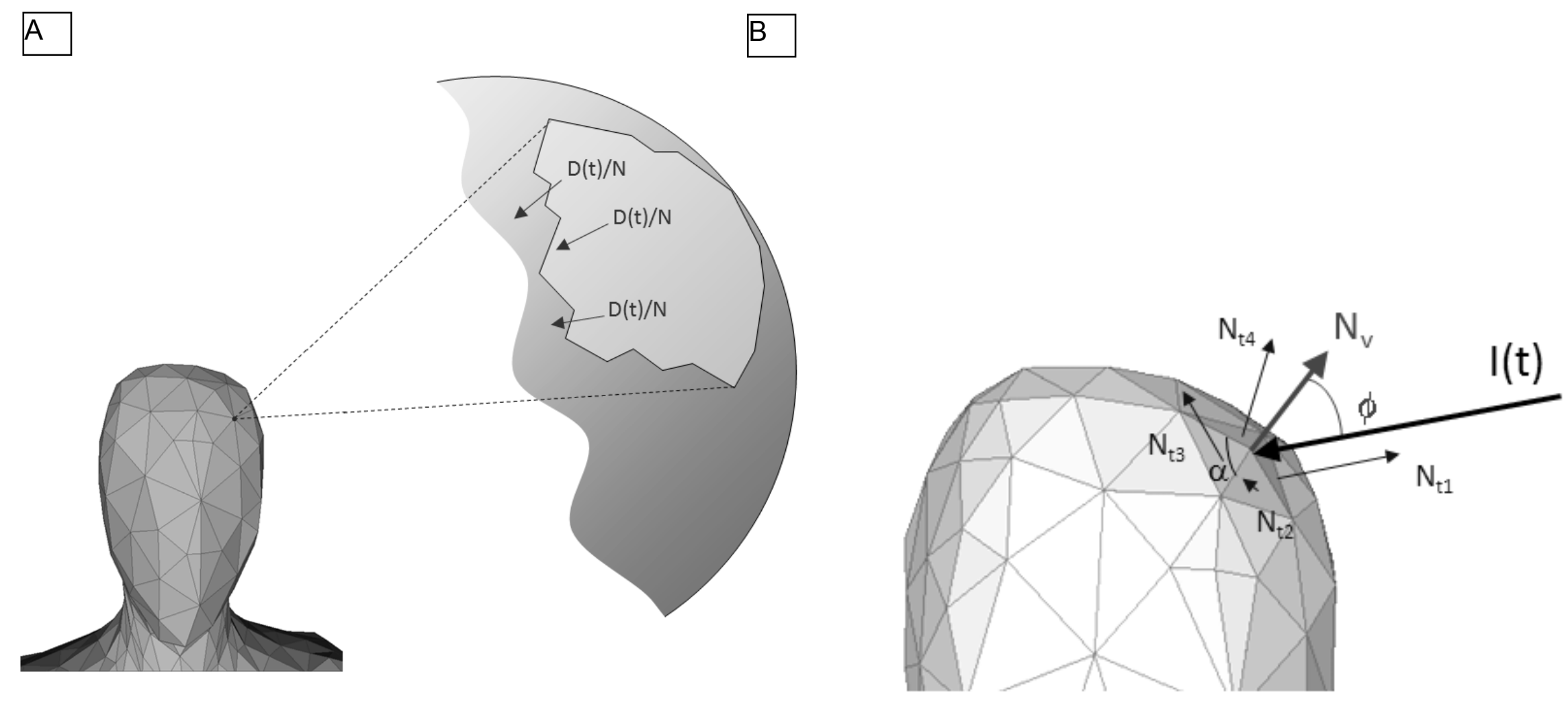
Figure 4. Measured vs. predicted daily exposure doses to solar UV $(n=54)$. Horizontal segments illustrate the $15 \%$ uncertainty on dosimetry. Hollow dots are outliers identified as measurement errors. 


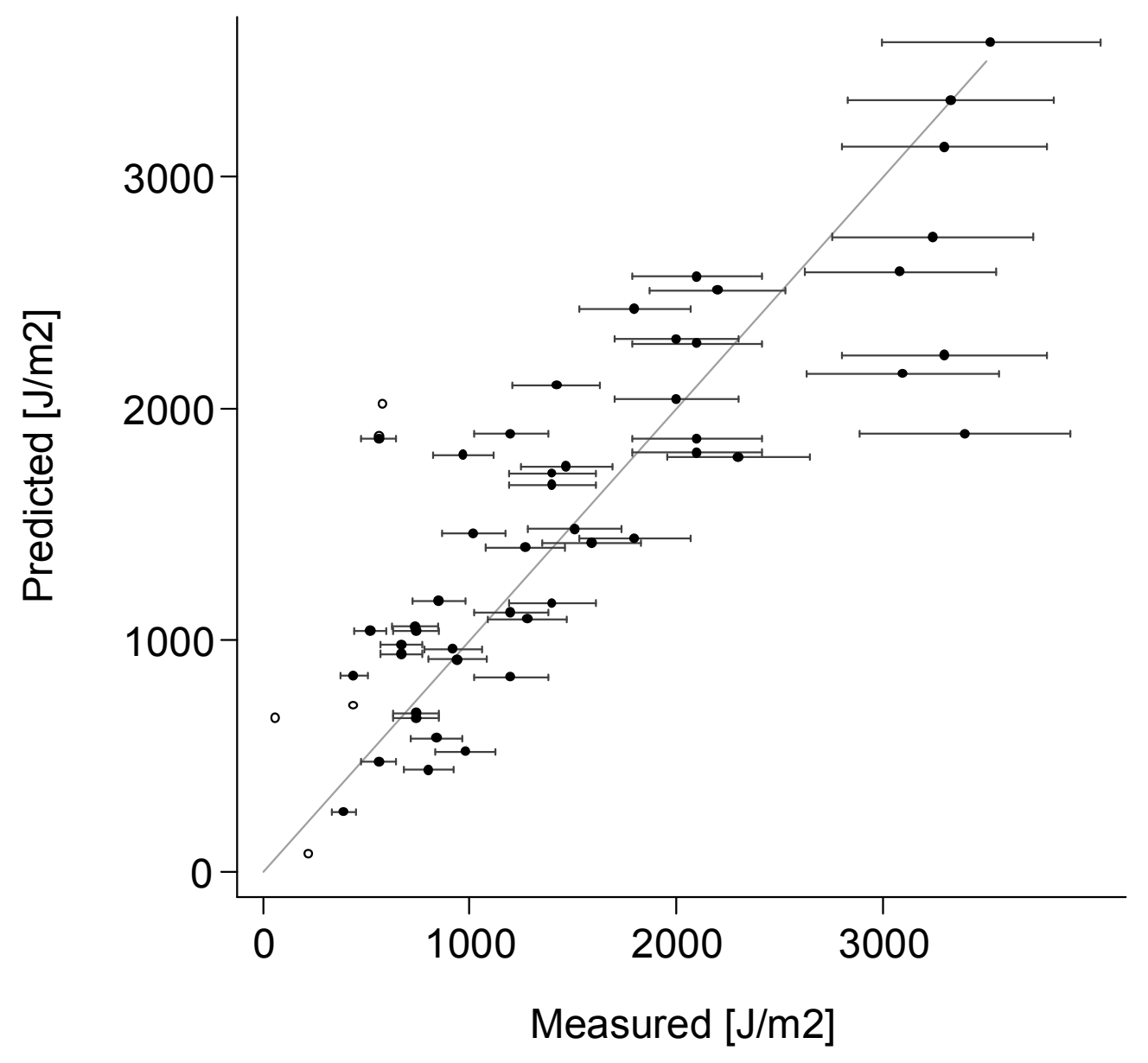


Figure 5. Predicted/Measured daily exposure doses to solar UV ( $n=49)$. Shaded area illustrates the dosimetric uncertainty (15\% relative error). Body parts are illustrated as such: $(\odot)$ neck, $(\square)$ low back, $(\Delta)$ left shoulder $(\diamond)$ right shoulder, $(\Delta)$ right wrist, $(\diamond)$ forehead, $(\square)$ top head, $(\circlearrowleft)$ chest and $(+)$ other body location. 


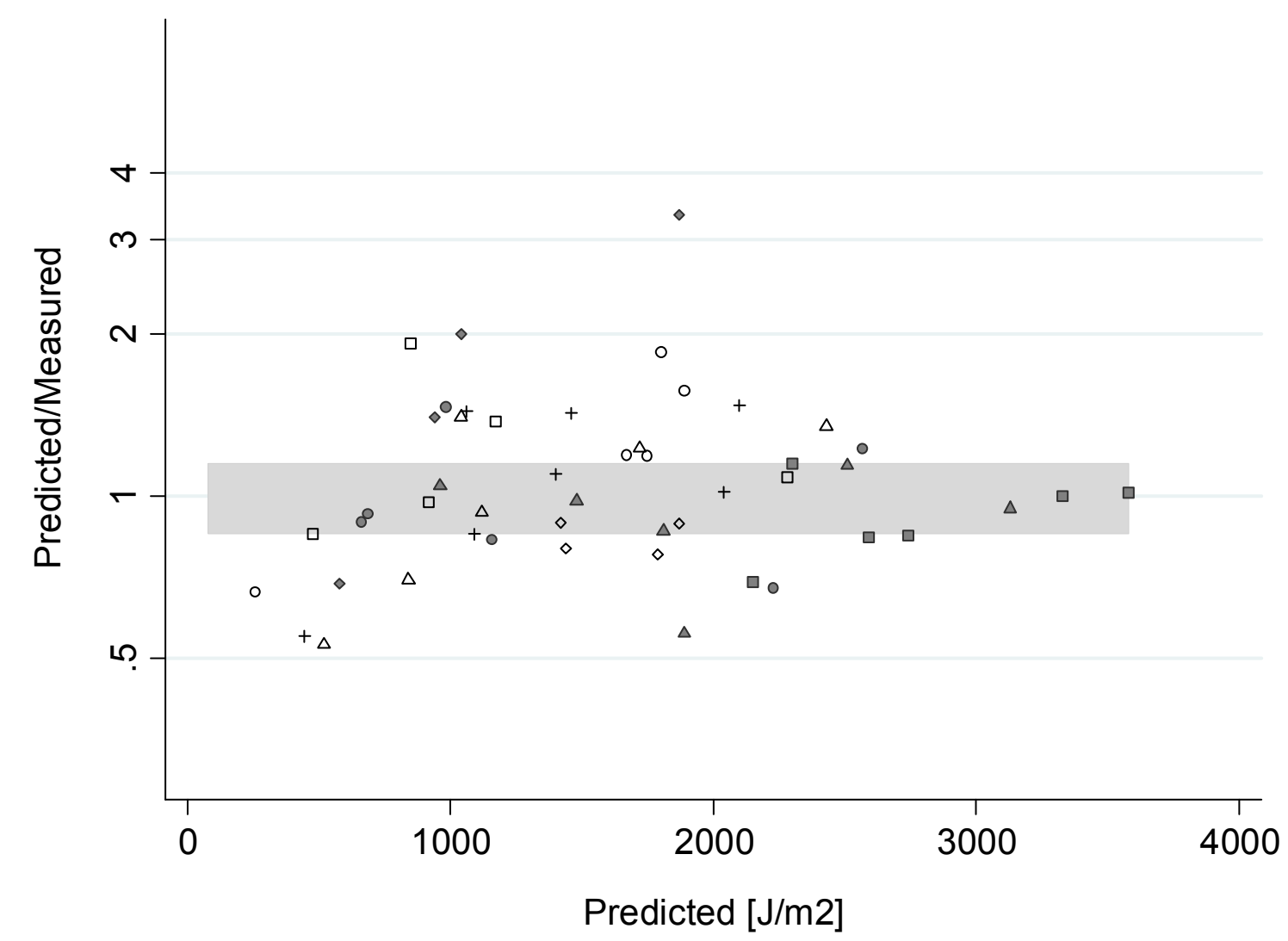


Figure 6. Visualizing simulation results for a daily exposure (A) standing erect arms down (B) kneeling (C) seated (D) seated with a changing orientation $\left(90^{\circ}\right.$ rotation every $30 \mathrm{~min}$.). Blue, green, red and white colors were attributed for 0-39\%, 40-78\%, $79-100 \%$ and >100\% of the ground irradiance respectively.. 


$$
|x| 1
$$

